\title{
Adaptive Learning Model Based on Ant Colony Algorithm
}

https://doi.org/10.3991/ijet.v14i01.9487

Rongxia Li $(\bowtie)$

Chongqing Technology and Business University, Chongqing, China

115084138@qq.com

\begin{abstract}
To better respond to people's demands for multimedia learning, appropriate learning paths should be offered based on their actual learning demands and different knowledge levels. Adaptive online learning model integrates and improves existing learning frameworks to offer a set of knowledge paths that can cater to differ7ent preferences, tastes, and knowledge levels of learners, no need for them to be aware of this. Based on the improved ant colony algorithm, an adaptive learning system model that can satisfy learners' demands is built herein with reference to the foraging approach of ants to traverse the paths, thereby to find the best learning path, while the classification method for some learning objects can determine the search parameters. This innovative approach proposed hereof can help improve learners' academic performance and learning efficiency.
\end{abstract}

Keywords-Ant algorithm, adaptive learning, learning path, learning style

\section{$1 \quad$ Introduction}

Along with the popularization of information technology, all kinds of new knowledge show an explosive growth, and people's thirst for knowledge is also unquenchable. In this context, online learning becomes increasingly important. Since various types of information may be distributed in different areas or held by different people, online education model should be developed to acquire diverse knowledge from each other. Online learning websites are highly flexible and interactive since they can help people save much time, reduce the transportation and exploration costs. But unfortunately, current online learning media can't provide different learners with the most applicable learning path, as they misfit well to their diverse demands of learners for knowledge. There is no best effect from these learning media since they seem underdeveloped and thus unpopular among the public. The most fit happens to offering tailored courses based on the learner's practical knowledge backgrounds and preferences. From this angle, online learning can be interpreted as a problem for searching for optimal paths. In this regard, many scholars have processed learners' data in line with their own learning capacity and style, to find the path most suitable for them. Scholars Felder et al. Believed that different students had unique learning styles of their own, and all students should not be assimilated against the development 
of them [1]. For example, some people can get ceiling efficiency when learning via the content visualization, and some can make a better experience by listening.

In Internet-based online learning, the learning content delivery should adapt to the learner's prior knowledge, learning speed, comprehension level, and so on. Early elearning platforms do not expand these features and present users with statically inflexible experience. The context-aware e-learning system provides learners with learning content based on what they are, where the context refers to any information of situation used to characterize entities as people, sites, and physical or computing objects. These parameters are used to generate a learning path that defines the sequence of activities that the learner performs with the units in the process of online learning. The units are abstracts for courses, seminars or any other formal or informal learning or pedagogical activities [2]. The challenge for online learning is how to choose the right learning content and provide it to learners. Curriculums must match the learner's acceptable level. Although many existing methods can generate learning paths, they fall short of backgrounds of all learners, such as profiles, infrastructure, preferences and learning background, etc. A learning schedule, therefore, must be designed to include any contexts of the learners in favor of them in the elicitation of most appropriate materials. There is only a learning path generation algorithm in the literature. We should develop an optimal algorithm for generating a learning scheme. This paper traces many existing learning models, and study one of the most suitable methods for students based on the ant colony algorithm. Here comes a new algorithm model.

\section{Pertinent study}

The adaptive education system is a new teaching method that attempts to replace the traditional non-personalized education model by offering the learner with proper materials. Its purpose is to maximize the subjective satisfaction of the learners. It also enables a real-time assessment on learning outcomes and progress [3]. Learner's feature recognition is the first phase of adaptive learning, called the modeling of learners. Based on the information collected in the first phase, the second phase of adaptive system is adaptive decision. The users must be modeled with a given number of parameters, which depends on prior demand surveys to identify learners' interests and difficulties, as the key to systemic adaptivity. As the peer scholars persevere with exploration of personalized education, especially in the wake of the Internet technology, this field has been developed dramatically.

Most online learning systems now use three individual parameters at most. These studies are mostly developed based on the learners' know-how, for example, PERSO employs a case-based reasoning that determines which appropriate courses should be recommended to learners based on their knowledge levels and preferences for these knowledge [4]. TANGOW is an adaptive learning system based on the FelderSilverman learning style model. It includes two dimensions, i.e. deduction/intuition dimension and sequence/global dimension. Learners are invited to fill in the learning style assessment form, and when they first interface to the system, the learning model 
will be initialized. The system will supervise and record learners' follow-up behaviors, based on which the model will be updated [5]. AHA is an education system based on the "educational meta-strategy", which specifies that the system's educators can choose the learning style published and the adaptive strategy. In this case, the system does not have a specific learning style. However, the system also imposes restriction on definable policy style, involving a set of available learning styles [6]. Another learning model VARK adopted by other educational systems mainly involves the perception form preferred for learning, that is, whether it is delivered by visual perception, or auditory perception, or other perception modes [7]. SAAD [8] aims to enable teaching content publishers to develop adaptive learning content based on the information system learning design, to ensure content interoperability, that is, interact with learners to improve themselves. Learner's knowledge level, learning language and perception mode are also adaptive. The WELSA as a network education system with learning style adaptability integrates several features of models proposed by other scholars to build an instructional system of unified learning model adaptable to the course learners [9].

\section{Ant colony algorithm and adaptive learning recommender architecture}

\subsection{Adaptive learning based on ant colony algorithm}

The ant colony optimization is a widely used heuristic algorithm where the "artificial ants" are applied to find the optimal path. Ant colony algorithms can solve a wide variety of problems using heuristic algorithms. This heuristic algorithm has great possibilities to solve problems and find better solutions. Moreover, this method can find a solution within an acceptable time for users. The typical problem solved using the ant colony algorithm is the Travel Salesman Problem (TSP) as typical one about optimal path. As shown in Algorithm 1 below, it is the first algorithm model proposed by Dorigo that is improved using ant colony optimization, and has been successfully applied. Formula 1 gives the mathematical model of ant colony algorithm [10].

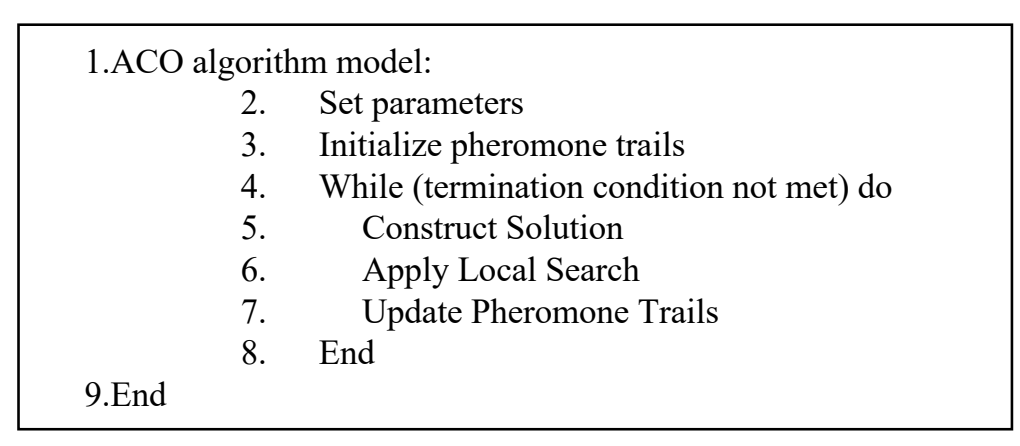

Algorithm 1: Ant colony optimization model 


$$
P_{i j}^{k}(t)=\frac{\left(\tau_{i j}(t)\right)^{\alpha}\left(\eta_{i j}(t)\right)^{\beta}}{\sum_{l \in N_{i}^{k}}\left(\tau_{i j}(t)\right)^{\alpha}\left(\eta_{i j}(t)\right)^{\beta}}, \forall j \in N_{i}^{k}, k \in 1 \sim m
$$

The algorithm applies the probabilistic behaviors to select the appropriate rule (called a random scale rule) for every procedure to determine the next node to be accessed. Specifically, it calculates the transition probability from current node $i$ to node $\mathrm{j}$, where gij $=1 / \mathrm{dij}$ is an available priori value. $\mathrm{a}$ and $\mathrm{b}$ are two parameters that determine the impact relative to the information path and the heuristic information. When nodes $\mathrm{i}$ and $\mathrm{k}$ belong to a set of nodes that have not been accessed (the probability that node Nki is chosen is 0 ), Nki is a feasible neighborhood of the algorithm. With this probability rule, the probability that the algorithm selects a specific arc $(i, j)$ will increase as the associated information path $\mathrm{Sij}$ and the heuristic information gij increase. The role of parameters $a$ and $b$ is to determine the calculation of the information trajectory and the heuristic path deviation.

The ant colony optimization is a meta heuristic method that usually solves the minimum cost problem. It marks the pheromone path to make it measurable. When there are more pheromones on the path, other paths may be used. Therefore, the path where there are pheromones will grow rapidly, and ultimately the area will be balanced overall. Similarly, learners will also have learning objects that are accessed most frequently in recent time. Ant colony optimization can use them to recommend appropriate learning materials. Therefore, adaptive learning based on ant colony algorithm can fully use the characteristics of learners and learning methods to provide the most appropriate learning objects in the learning system.

\subsection{Architecture of adaptive learning system}

As shown in Fig. 1, it is the architecture of an adaptive learning system based on ant colony algorithm. The system includes learners, e-learning system, learner database, learning object database, and authors and algorithms for adaptive learning based on ant colony optimization. At the outset, learners' access to the e-learning system. The learners' profiles, such as learning style, knowledge level, and search keywords, will be automatically updated in the learner database.

These profiles will be analyzed by ACO-based adaptive learning algorithm. It also provides a heuristic matching rule that takes charge of arranging the learning objects and calculating the matching rate. The author uploads the new learning objects to the Learning Object (LO) database in a general format so that learners can efficiently search and retrieve the appropriate learning objects.

The architecture covers various learning skills and objects. With this system, any learning object, if appropriate, can be easily recommended to the users. In the above system architecture, the user needs to register itself and create complete profiles, including learning style and current learning level, which can help the system identify whether users are beginners or have intermediate or advanced expertise. If the user logs in the system for the first time, after the registration, the current system has no history of his or her searches. 
However, when his login time and searches for relevant learning objects progressively increase, the system stores the user's history data for recommending thematic learning objects for the most recent search of users next time. During the operation of the system, the user only needs to enter the keywords for search, and then apply the distance and heuristic algorithms to the search. In the end, those paths with the shortest distance and matching rules will be displayed to the user to meet the user's diversified demands for learning but not perceived by users.

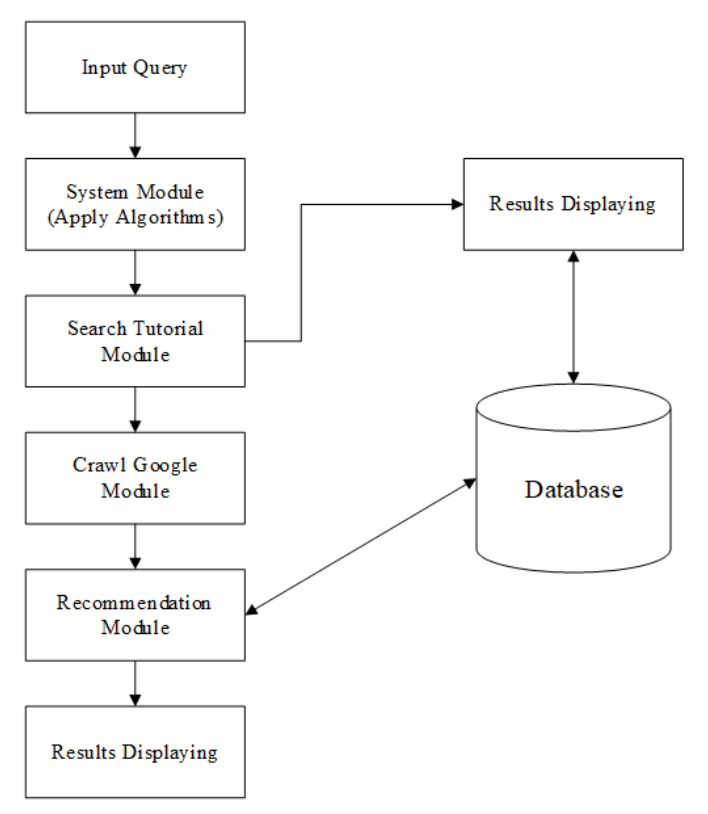

Fig. 1. Architecture of adaptive learning system based on ACA

\subsection{Building the ALS architecture}

The pseudo code of the attribute-based ant colony system proposed in this paper is shown in Algorithm 2, which can be implemented in different programming languages, so that it has a strong transportability. 


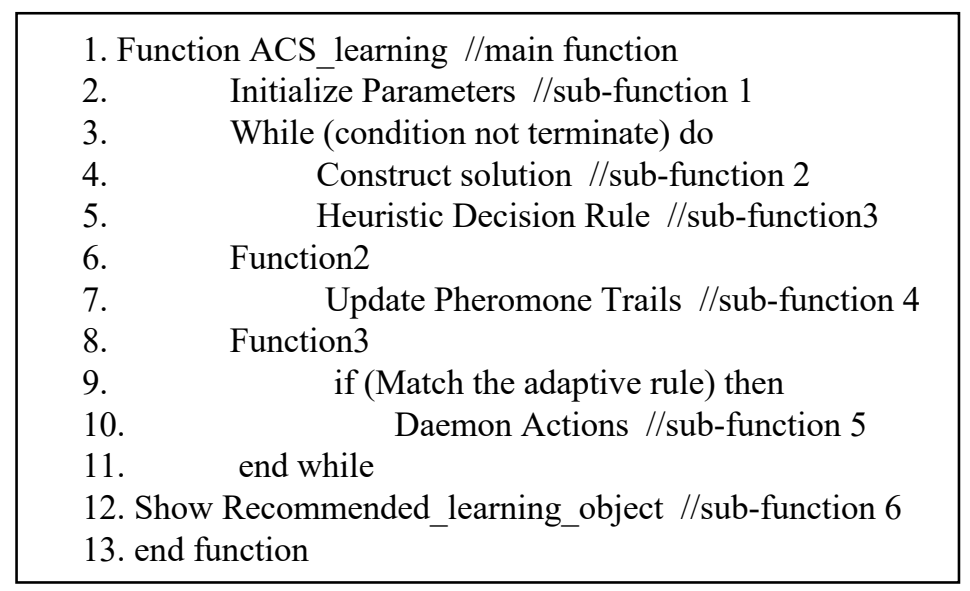

Algorithm 2: Pseudo code of attribute-based ant colony adaptive learning system

To build this adaptive learning system, there are five modules needed to be built. Among them, Module 1 is the system module. In this module, the system view is designed with proper login authentication in the system. Module 2 is to add a learning object module whose role is to add learning objects to the database. Module 3 is a search course module, which is mainly divided into two sub-modules, i.e. the ant colony algorithm module, which is mainly used to search a database queried by users using an ant colony optimization; the database service module, which adds learning objects to the database and gets the courses that the users desire from the database. Module 4 is the Google crawler module. If the ant colony adaptive system does not find any relevant courses, the module will search for the same query on Google and display the results it hits. This process is completely transparent to the user.

Therefore, they will think it as learning system course to enhance the user's recognition of the system. Module 5 is a recommender module, used to provide recommended search results to the user according to users' previous search history. This module is customer-oriented, so it must be user-friendly so that all functions of the whole system's backend are fully recognized by the users to reach the ultimate destination.

The adaptive learning system based on ant colony optimization has a high performance and can meet the diversified demands of most learners. First, it does not need to guess the knowledge and preferences that users desire, as what the previous online learning systems have done, but to accurately push them based on user's own data [11]. Second, as users use the ant colony optimization, the system can quickly respond to the user's educational demands. Instead of spending hours to read a huge mass of unwanted data for searching the appropriate resources, users can quickly get the resources or appeals they desire about education issues [12].

Moreover, as mobile smart devices grow in popularity, the system can meet the educational demands of users where and when they want [13]. Most e-learning systems are now accessed at any time via the Internet. This system will provide unique register 
and login information for each user and will tail after their learning process. Over time, more and more user data will be stored on the system, and can be processed by more intelligent data analysis technology or artificial intelligence technology, such as genetic algorithm, SVM, Tensor Flow and other algorithms and technologies. These new technologies will gradually improve their precise push [14-17]. These measures will help understand the diverse behaviors of learners, providing important assistance for learners' behaviors and preferences, and motivating them to offer more useful information. The last point to be explained is that the system will be able to determine what is the learning mode of the learner. The ACA-based adaptive e-learning system can continuously track down the user's learning situation, and make a comprehensive evaluation based on the information and the latest learning profile, to find the currently optimal learning path and adjust it to achieve the best learning effect.

Although the adaptive learning system based on ACA can greatly help learners to learn quickly, in today's fast-paced educational situations, many learners do not have enough patience to wait and accomplish the learning objects recommended by adaptive learning system recommender. Many learners are mostly eager for quick success. Although the system can offer good materials to help students for learning, it is difficult to change the psychological habits of learners. This is also impossible technologically, so that human intervention is required to achieve the best learning effects.

\section{Conclusion}

This paper proposes an adaptive learning model based on ant colony algorithm, by which adaptive learning platform system can be achieved. Algorithm model blends users' personalized information, including personal education background and learning style. Additionally, an adaptive learning system based on ant colony algorithm can help teachers develop personalized courses for different students and provide appropriate learning objects for learners, which, on the one hand, can improve the learning environment of students and serve them to learn better; on the other hand, this is also an improvement of the existing education system, which contributes to explore better quality education services.

Ant colony algorithm is an excellent way that can solve combinatorial problems. Sharing data on pheromones is a highlight of the algorithm. It helps enrich the knowledge of the overall path each search. By implementing the system, learners will be contributed with rich knowledge in relevant fields and learning objects, as provided with an adaptive solution. This algorithm model needs to optimize all parameters to build up the most appropriate online learning system. The implementation of adaptive learning system especially applies to learners who do not have self-learning competence. With it, multiple algorithms can be applied to generate better learning paths, improve the algorithm model and its precise. In the future work, the algorithm model will be implemented in detail, and the theory will be implemented in available systems to meet the actual demands the majority of learners. 


\section{$5 \quad$ References}

[1] Felder, R. M., \& Silverman, L. K. (1988). Learning and teaching styles in engineering education. Engineering education, 78(7), 674-681.

[2] Tuparova, D. \& Tuparov, G. (2006). Learning paths in open source e-learning environments. Current Developments in Technology-Assisted Education, 1565-1569.

[3] Midgley, C. (2014). Goals, goal structures, and patterns of adaptive learning, Routledge. https://doi.org/10.4324/9781410602152

[4] Chorfi, H., \& Jemni, M. (2004). PERSO: Towards an adaptive e-learning system. Journal of Interactive Learning Research, 15(4), 433-447.

[5] Paredes, P., \& Rodriguez, P. (2004). A mixed approach to modelling learning styles in adaptive educational hypermedia. Advanced Technology for Learning, 1(4), 210-215. https://doi.org/10.2316/Journal.208.2004.4.208-0823

[6] Stash, N. (2007). Incorporating cognitive/learning styles in a general-purpose adaptive hypermedia system. Dissertation Abstracts International, 68(04). https://doi.org/10.1145/13 24960.1324963

[7] Prithishkumar, I. J., \& Michael, S. A. (2014). Understanding your student: using the VARK model. Journal of postgraduate medicine, 60(2), 183186.https://doi.org/10.4103/0022-3859.132337

[8] Bourbia, R., Seridi, H., \& Seridi, A. (2008). SAAD: An adaptive e-learning platform using IMS Learning Design. Academy of Management Learning \& Education, 14(1), 142.

[9] Popescu, E., Trigano, P., Badica, C., Butoi, B., \& Duica, M. (2008). A course authoring tool for WELSA adaptive educational system. Proc. ICCC 2008, 531-534.

[10] Liu, H.H., Li, N. (2017). Application of self-adaptive ant colony algorithm in machine technology manufacturing process route optimization, Academic Journal of Manufacturing Engineering, 15(1), 11-16.

[11] Driscoll, M. (2010). Web-based training: Creating e-learning experiences. John Wiley \& Sons.

[12] Shor, I. (2012). Empowering education: Critical teaching for social change. University of Chicago Press.

[13] Lehner, F., \& Nosekabel, H. (2002). The role of mobile devices in E-Learning first experiences with a wireless E-Learning environment. In Wireless and Mobile Technologies in Education, 2002. Proceedings. IEEE International Workshop on IEEE, 103-106.

[14] Castro, F., Vellido, A., Nebot, À., \& Mugica, F. (2007). Applying data mining techniques to e-learning problems. In Evolution of teaching and learning paradigms in intelligent environment, Springer, Berlin, Heidelberg, 62, 183-221. https://doi.org/10.1007/978-3-54071974-8 8

[15] Asim, I., Muhammad, S., Robert, A. (2018). Cyber security in internet of things, Review of Computer Engineering Studies, 5(1), 17-22.

[16] Isah, OR, Usman, AD, Tekanyi, AMS. (2017). A hybrid model of PSO algorithm and artificial neural network for automatic follicle classification. International Journal Bioautomation, 21(1): 43-58.

[17] Ghritlahre, H.K., Prasad, R.K. (2018). Investigation on heat transfer characteristics of roughened solar air heater using ANN technique, International Journal of Heat and Technology, 36(1), 102-110. 
Paper-Adaptive Learning Model Based on Ant Colony Algorithm

\section{Authors}

Rongxia Li, female (1984.06--), Master, Modern International Academy of design art, Chongqing Technology and Business University, Chongqing 401120, China. The main research areas: ideological and political education and pedagogy, E-mail: 115084138@,qq.com

Article submitted 04 August 2018. Resubmitted 04 September 2018. Final acceptance 14 October 20187. Final version published as submitted by the authors. 